\title{
A Deep Learning Based Method for Cutting Parameter Optimization for Band Saw Machine
}

\author{
Pin $\mathrm{Li}^{1}$, Jianshe Feng ${ }^{2}$, Feng Zhu ${ }^{3}$, Hossein Davari ${ }^{4}$, Liang-Yu Chen ${ }^{5}$, Jay Lee ${ }^{6}$ \\ 1,2,3,4,6 NSF I/UCR Center for Intelligent Maintenance Systems, Department of Mechanical Engineering, University of Cincinnati, \\ Cincinnati, Ohio 45221-0072, USA \\ lip2@mail.uc.edu \\ fengje@mail.uc.edu \\ zhufn@mail.uc.edu \\ davarihn@mail.uc.edu \\ jay.lee@.uc.edu \\ ${ }^{5}$ COSEN Mechatronics CO. LTD, No. 309, Sec. 2, Houke Rd., Houli Dist., Taichung City 421, Taiwan (R.O.C.) \\ liang_yu@cosen.com.tw
}

\begin{abstract}
Throughout the rough machining stage, band saw machines are widely used to cut various raw materials into the required dimensions. The replacement of a blade due to the blades degradation accounts for a large part of the total cost of band saw machine usage. Therefore, mitigating the blade degradation by dynamically optimizing the cutting parameters can produce great economic benefit and can also be a leading factor toward realizing smart manufacturing. This paper proposes the steps to achieve this goal, starting with applying the Convolutional Neural Network (CNN) model to map the intricate relationship between the cutting parameters and blade degradation. Next, a simulation technique is used to find the optimal cutting parameters based on the blade degradation estimation from the model output, which can extend the blade life. The proposed optimization method is then validated on the data collected during a real manufacturing process using band saw machines. The comparative results demonstrate that the obtained optimal cutting parameters can effectively extend the service life of the blade for a band saw machine.
\end{abstract}

\section{INTRODUCTION}

In the rough machining stage, band saw machines are widely used to cut various raw materials into the required dimensions. Currently, the band saw machine is one of the most frequently used machine tools, being used on metal, wood, plastic material and even in a semiconductor chip. Pin Li et al. This is an open-access article distributed under the terms of the Creative Commons Attribution 3.0 United States License, which permits unrestricted use, distribution, and reproduction in any medium, provided the original author and source are credited.
There are several issues that need to be addressed and solved throughout the use of a band saw machine, including ban saw tracking, vibration, etc. The selection of optimal cutting parameters, such as blade speed and downward speed, is one of the most vital issues, as improper selection of parameters may result in (1) excess vibration of the blade caused by the higher cutting parameter, leading to abnormal wear of band saw blade, (2) low sawing efficiency for the lower cutting parameter. Currently, the cutting parameters are determined based on the operator's experience, which can potentially cause two major issues: (1) experience is subjective, which can lead to non-consistent cutting performance among different operators, especially for beginners; (2) experience cannot provide quantitative evidence to guide future improvements. Moreover, the empirical parameters selection will most likely not take into account all the conditions in real production.

The problem of cutting parameter optimization has been extensively investigated in machining application. Current parameter optimization methods can be classified into two categories: (1) physics-based and (2) data-driven methods. For physics-based methods, Bhattacharya (Bhattacharya, Das, Majumder, \& Batish, 2009) presented an experimental study to investigate the effects of cutting parameters on finished product as well as power consumption. To do this investigation, Bhattacharya employed Taguchi techniques, finding that cutting speed has a significant effect on the surface roughness and power consumption, while other parameters did not. Camposeco-Negrete (CamposecoNegrete, 2015) built a regression model for multiple response variables by using a Central Composite Design and the Response Surface Method, finding the relationship between cutting parameters and the response variables and also 
defining the values of the variables that achieved the optimal objective. Sarikaya (Sarikaya \& Güllü, 2015) presented an approach for optimization of machining parameters with multi-response outputs, including flank wear, notch wear, and surface roughness. The process parameters, including cutting fluid, fluid flow rate and cutting speed, were simultaneously optimized by Taguchi based grey relational analysis. Data-driven predictive modeling methods that were built upon statistical methods or machine learning algorithms(Jia, Di, et al., 2018; Jia, Jin, et al., 2018; Lughofer \& Pratama, 2018; Sangwan, Saxena, \& Kant, 2015) proposed an integrated Artificial Neural Network-Genetic Algorithm (ANN-GA) approach to determine the optimal machining parameters, leading to minimum surface roughness which is widely used as an indicator of product quality.

Coversely, in current literature, most of the research focuses on improving energy efficiency in specific material application areas. Few investigations are found for optimal parameters based on equipment degradation. In addition, there are currently few published papers that focus on parameter optimization of the band saw machine. Saglam (Saglam, 2011) applied Taguchi's approach along with Artificial Nerual Network (ANN) to estimate teeth wear of band saw blade. In this study, they considered speed, feed, cutting length and material hardness as input parameters and investigated the effect of these parameters on blade wear. Asiltürk (Asiltürk \& Ünüvar, 2009) proposed a neural-fuzzybased force model for controlling the band sawing process, and cutting parameters were continuously updated by another neural network. Mewada (Mewada \& Raval, 2016) combined Taguchi and Desirability function for optimization of the multi-response objective. There is currently no literature or research using deep learning for optimization of cutting parameter in the band saw machine.

To address the issues mentioned above, we propose a deep learning based approach to find optimal parameters based on blade degradation, which would prolong the life of band saw blade. Deep learning has been proven to be successful in a wide range of applications (Jing, Zhao, $\mathrm{Li}, \& \mathrm{Xu}, 2017$ ) due to its powerful learning ability, which automatically learns features from raw data without human intervention (J. Li, Mei, Prokhorov, \& Tao, 2017). One essential advantage of the proposed method is that it takes the advantage of deep leanring to find the complicated relationship between the cutting parameters and degradation of the band saw blade. Also, we considered combining expert knowledge for parameter optimization. The objective is to increase production efficiency while reducing band saw blade wear. Based on the above considerations, the proposed framework includes two main parts: (1) building a model to generate the health index for band saw blade during cutting, (2) mapping the relationship between the cutting parameters and the band saw blade degradation based on the Convolutional Neural Network (CNN) model, which is then used to search the optimal cutting parameters.
The remainder of this paper is organized as follow: Sections 2 introduces the data acquisition system and related work in establishing the health index for blade, section 3 presents the proposed methodology for cutting parameter optimization, the verification results are discussed in section 4 , and conclusion and future work are provided in section 5 .

\section{RELATED WORK}

\subsection{Experiment and Data Acquisition}

An experiment with band saw machines provided by Cosen Mechatronics, as shown in Figure 1, was employed for data acquisition. The band saw machine was set up with accelerometers, microphones, thermal couples and pressure gauges installed near the blade guide. For each machine, the add-on sensors and the PLC controller are connected to computer for data acquisition, management, and analysis. In this experimental investigation, three major data sources are collected including cutting task specifications, cutting parameters and measured process variables. The cutting task specifications are task-oriented, which are related to machine types, blade choices, and material properties. Two cutting parameters including blade speed and downward speed are parameters considered important in our study. Measured process signals include vibrations in the cutting area, temperatures of critical components, etc. The vibration data is collected with a sampling rate of $10.24 \mathrm{kHz}$, which is used to evaluate the performance of the cutting processes. The sampling rate of other signals is set as $100 \mathrm{~Hz}$, but the mean values of these signals in every 2 minutes window are calculated and recorded. The details of the collected signals can be found in

Table 1.

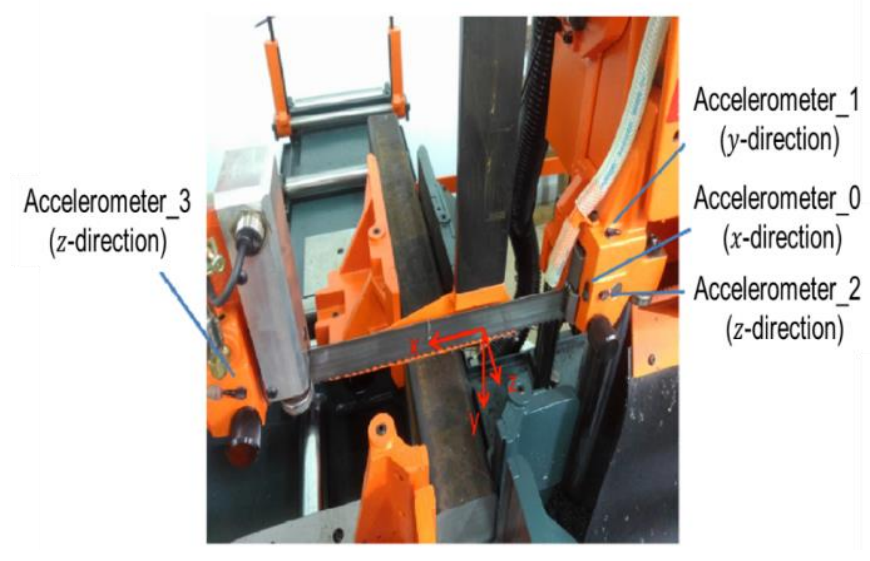

Figure 1. Band saw machine with accelerometers. 
Table 1. List of collected data.

\begin{tabular}{|c|c|c|c|}
\hline Variable & Variable name & Data type & Physical meaning \\
\hline \multirow{9}{*}{$\begin{array}{l}\text { Cutting Task } \\
\text { Specification }\end{array}$} & Machine ID & Discrete & The id number of band saw machine \\
\hline & Blade ID & Discrete & The id number of blade \\
\hline & Blade TPI & Discrete & Tooth per inch for blade \\
\hline & Blade type & Discrete & Specific type of band saw blade \\
\hline & Blade brand & Discrete & The brand of blade used \\
\hline & Blade material & Discrete & Basis material of blade \\
\hline & Cutting material & Discrete & The material type of cutting work piece \\
\hline & Material height & Discrete & The height of cutting work piece \\
\hline & Material width & Discrete & The width of cutting work piece \\
\hline \multirow{2}{*}{$\begin{array}{l}\text { Cutting } \\
\text { parameter }\end{array}$} & Blade speed & Continuous & $\begin{array}{l}\text { The speed at which the blade rotates, unit is } \\
\mathrm{m} / \mathrm{min}\end{array}$ \\
\hline & Downward speed & Continuous & $\begin{array}{l}\text { The speed at which the blade move through the } \\
\text { material being cut, unit is } \mathrm{mm} / \mathrm{min}\end{array}$ \\
\hline \multirow{11}{*}{$\begin{array}{l}\text { Measured } \\
\text { process variable }\end{array}$} & Cut number & Continuous & Number of cut for one blade \\
\hline & Accumulated area & Continuous & Accumulated area of cut work piece \\
\hline & Flow speed & Continuous & Flow speed of coolant \\
\hline & Flow PH value & Continuous & PH value of coolant \\
\hline & Main motor current & Continuous & Measures the torque of motor \\
\hline & Accelerator & Continuous & Measures the vibration \\
\hline & Blade deviation & Continuous & Measures the blade bent content \\
\hline & Ambient temperature & Continuous & temperature of ambient environment \\
\hline & Coolant temperature & Continuous & Temperature of coolant temperature \\
\hline & Hydraulic temperature & Continuous & Temperature of hydraulic system \\
\hline & Gearbox temperature & Continuous & Temperature of gearbox \\
\hline
\end{tabular}

\subsection{Health Index Construction}

The Health Index (HI) construction for machines are well studied in the area of PHM. Typically, the HI for machines or critical components is built from the measured data using signal processing techniques, machine learning and data mining techniques to quantify the health status at different times (P. Li et al., 2018) (Jia, Zhao, Di, Li, \& Lee, 2018) (Jia, Huang, Feng, Cai, \& Lee, 2018) (Qiao et al., 2019) (P. Li et al., 2019). In our study, the vibration data is mainly adopted to build the HI model to present the blade degradation process. Every two minutes, a set of feature values are extracted from the vibration data acquired in this time interval including RMS, crest factor, peak to peak value and octave frequency band energy of vibration signals. By using the Fisher Criterion, we select the top 10 features by comparing each feature changes in values from early life to end of life . The final system health measurement $y_{k}$ is the average of the top 10 features from vibration signals, as shown in Eq. (1).

$$
y_{k}=\frac{1}{10} \sum_{\mathrm{j}=1}^{10} y_{k}^{j}
$$

Where $y_{k}^{j}$ is the selected $j$ th important feature. In this way, the multi-dimensional features are fused into a single variable $y_{k}$ as the final system HI. More details can be found in our previous work (Yang, 2016).

\section{PROPOSED Methodology}

\subsection{Overview}

The traditional workflow of cutting includes the following steps. First, the cutting task is given, such as specification of cutting blade, material and size of the work piece. Next, the operator sets up the cutting parameter based on guidebook instruction and their experience. Thirdly, the operator checks the quality of the cut product and if surface quality is not good, the operator will need to discard the piece or recut it by adjusting the cutting parameters. Human expertise plays a significant role throughout the entire process and cannot simply be automated.

As shown in Figure 2, the contribution of this research is the development of the adaptive cutting parameter optimization module to enhance the traditional cutting workflow based on collected data. The developed module mainly contains three parts, which can dynamically adjust the cutting parameter 
during the cutting process. The basic idea of optimization is to find the optimal solution by evaluating all possible options. Therefore, the first step is to generate a comprehensive library of the possible cutting parameter pairs. The second step is to evaluate the goodness of each pair according to a designed objective function. The third step is to find the optimal cutting parameter pair considering both the objective function and cutting efficiency. Details of each step are introduced in the following sections.

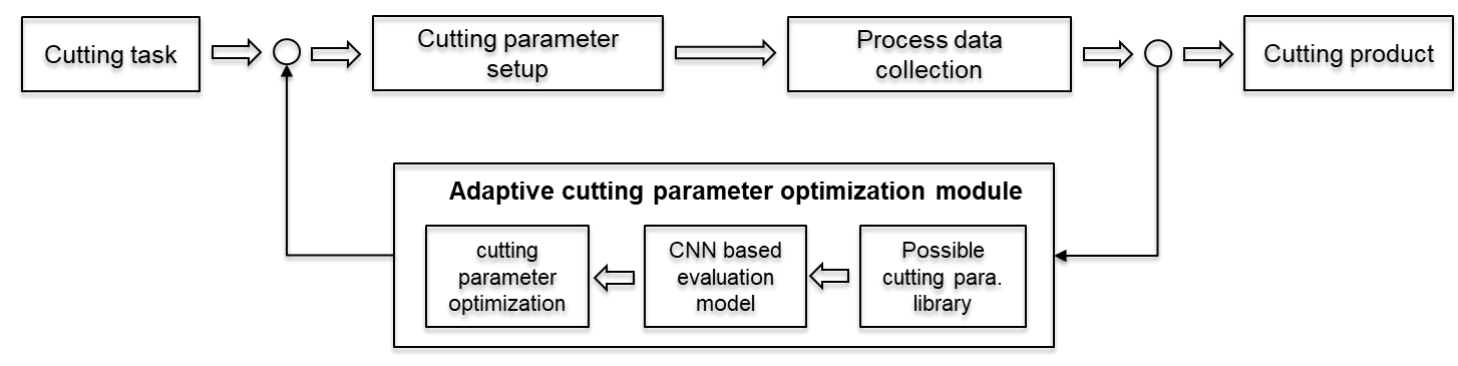

Figure 2. Flowchart of proposed methodology.

\subsection{Construct the Library of Possible Cutting Parameter Pairs}

Given the information of the cutting blade, material and size of work piece, the machine operator is able to get a rough range for cutting parameters from the instruction guidebook. In practice, the operator usually selects one specific value for the cutting parameter based on their experience. However, in this research, all values with fixed small interval within the range are used to construct the library. For example, if the retrieved value range for blade speed is [89, 121], and the interval is set as 1 , then the possible candidate would be [89, $90,91, \ldots, 120,121]$. As a first step, all combinations of blade speed and downward speed are evaluated based on their feasibility using the MORSE formula, as shown in Eq. (2). The MORSE formula is an empirical criterion to guarantee the desired chip load for the band saw blade.

\section{downward speed$$
=\text { blade TPI } \times 39.37 \times \text { blade speed }
$$$$
\times \text { blade tooth bite }
$$

Where the blade TPI represents the number of tooth per inch of the blade, which is a scalar value without unit. Blade tooth bite, also known as chip load per tooth, is the desired penetration of each tooth into the work piece. The safe and efficient range for tooth bite is [0.005, 0.01].

It is noted from Eq. (2) that the tooth bite can be calculated using downward speed and blade speed, and if the calculated value is beyond the safe range, the combination of blade speed and downward speed should be discarded. Therefore, the MORSE formula is used to filter out unreasonable cutting parameter pairs for the constructed library.

\subsection{CNN Based Evaluation Model}

Convolutional Neural Network (CNN) is one of the most popular deep learning algorithms. Due to its powerful capability of information representation, CNN has been widely used for feature extraction in many areas, especially in image processing. Different from back propagation neural network, CNN is a sparsely connected neural network consisting of three different types of layers: convolutional layer, pooling layer, and fully connected layer. The convolutional layer can have several different filters which can convolve on partial of the input layer to extract different types of features. The pooling layer is used to reduce the dimension of the extracted feature map and ensure the retained features are invariant to scale and orientation changes. The fully connected layer is used to bridge the extracted features and the target output. For an output with discrete values, softmax layer is utilized and for an output with continuous values, regression layer is used.

The health index is already established to monitor the degradation of blade based on the vibration signal, with the larger values of the health index indicating more degraded blades. Therefore, the health index can be treated as an objective function to evaluate the goodness of the cutting parameters. The CNN model is utilized to map the complex relationship between the cutting parameter, collected process variable and the established health index. Therefore, the possible cutting parameter pairs from the constructed library can be evaluated based on the output of the CNN model.

In section 2 , it is noted that there are three types of variables, which are recorded every two minutes. The blade health index is calculated every two minutes as well. All variables in

Table 1 with the exception of vibration and machine ID are organized parallel to construct a 2-dimensional input for the 2D CNN. The sliding window with a length 20 and stride 1 is utilized to truncate the data into small blocks. Therefore, the input layer receives a $20 \times 20$ data matrix. 


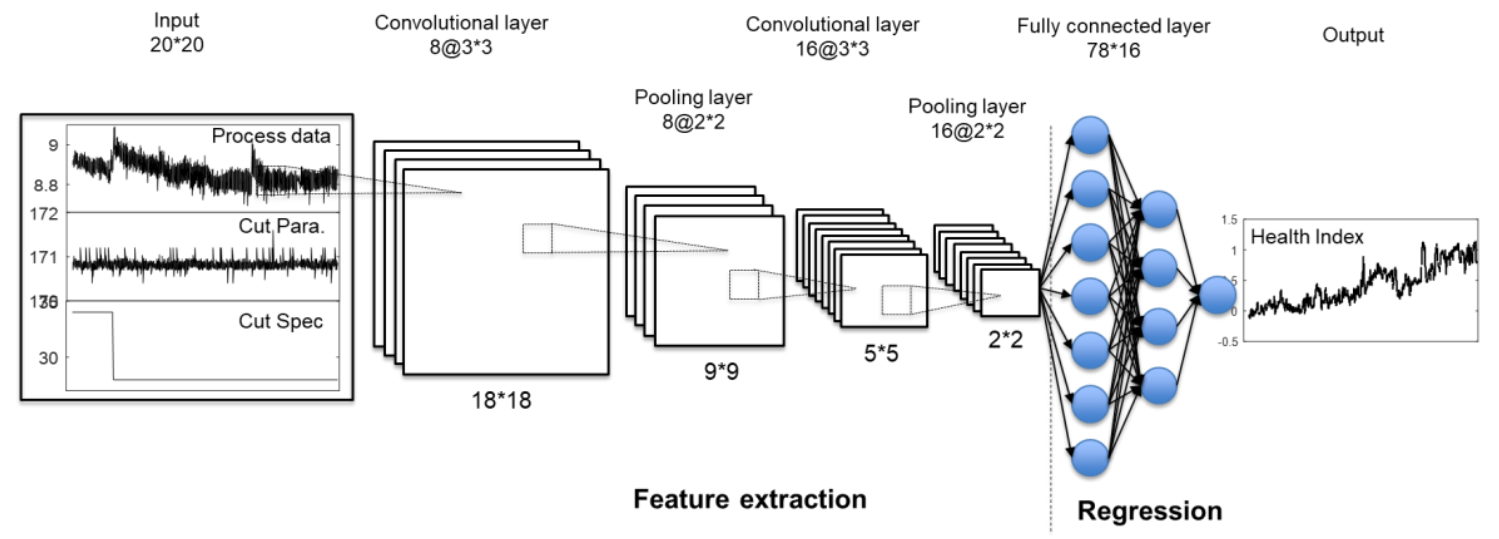

Figure 3. Structure and specification of CNN model.

Next, the two convolutional layers and two pooling layers are stacked followed by fully connected layers for regression purpose. The target output is the estimated health index, which is used as objective function to evaluate the goodness of each possible cutting parameter pair. The max pooling mechanism is used for each pooling layer. The number and size of each filter as well as the dimension of each layer can be seen in Figure 3.

In order to demonstrate the effectiveness of the CNN based regression, three traditional machine learning regression models are utilized in this research work for comparison. These regression models are Support Vector Regression with RBF kernel, Random Forest, and Neural Network. Truncation of data via sliding window is not applied for these regression models since they receive 1-dimentional input. Here, two metrics including root mean square error (RMSE), and mean absolute error (MAE), as shown in Eq. (3) and Eq. (4), are used to evaluate the performance of the regression models.

$$
\begin{aligned}
& \text { RMSE }=\sqrt{\frac{1}{N} \sum_{i=1}^{N}\left(\widetilde{y_{l}}-y_{i}\right)^{2}} \\
& \text { MAE }=\frac{1}{N} \sum_{I=1}^{N}\left|\widetilde{y}_{l}-y_{i}\right|
\end{aligned}
$$

Where $y_{i}$ and $\widetilde{y}_{l}$ are the true and predicted blade HI.

\subsection{Cutting Parameter Optimization}

At each time, when the cutting task is specified, all possible cutting parameter pairs are stored in the library and then evaluated by the CNN based model. Since a higher cutting rate is always favored by the machine operator, the cutting parameter pairs with higher downward speed is preferred over simply choosing the cutting parameter with minimal predicted health index value. As shown in Figure 4, all possible cutting parameter pairs are fed into the CNN model to obtain the predicted health index (HI), then the $10 \%$ quantile of all predicted $\mathrm{HI}$ is calculated as a criterion to retain the most promising cutting parameter pairs. Since the downward speed is closely related to the cutting rate, the cutting parameter with highest downward speed is chosen as optimal cutting parameter pair.

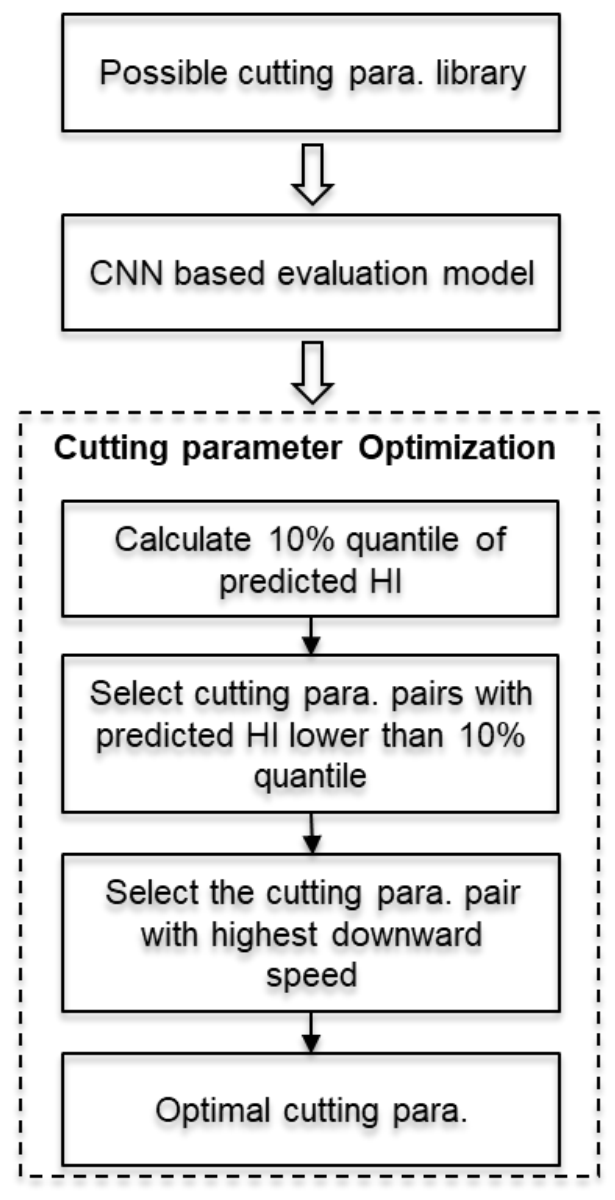


Figure 4. Flowchart of cutting paramter optimization.

\section{RESULTS AND DISCUSSION}

\subsection{Evaluation of CNN Model}

Data from 9 blades are collected for analysis in this research. Data from 8 blades are used to train the CNN model and the data from one blade is used for testing. In order to reduce the randomness of the results, all 4 models are tested ten times, and the average of the evaluation metrics are shown in Table 2. In addition, the regression performance of all models are visualized in Figure 5. It is noted that the developed CNN model performs best among those four models, as it's able to

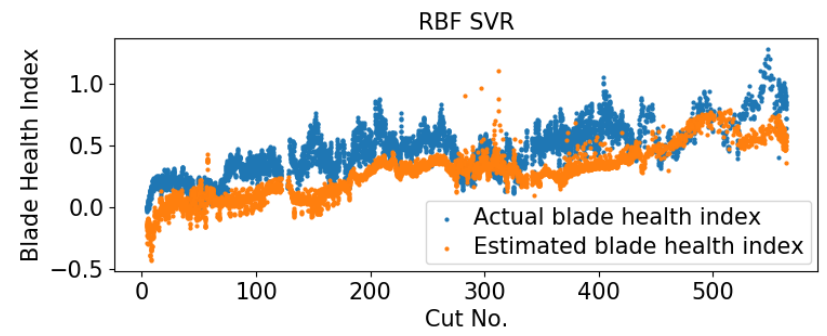

(a)

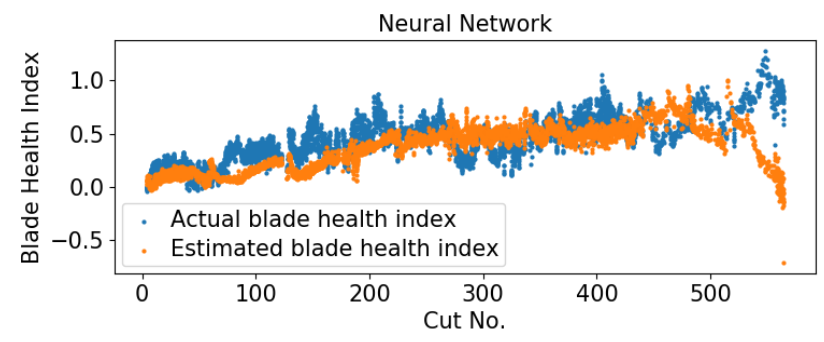

(c)

Figure 5. Regression result of all compared methods: (a) RBF SVR, (b) Random Forest, (c) Neural Network, (d) CNN.

\subsection{Evaluation of the Cutting Parameter Optimization}

The same testing blade data is used to demonstrate the result of the cutting parameter optimization. In practice, the cutting parameter would remain the same for a long time and the blade health index would change slightly over a short time period. Therefore, in this study, the cutting parameters are dynamically adjusted every 50 cuts, as shown in Figure 6(ab). The optimized health index is then compared with the actual blade health index, as shown in Figure 6(c). It's noted that the optimized health index is lower than the actual health index, which means the cutting parameter optimization could prolong the blade lifespan.

\section{CONCLUSION}

This paper proposed a deep learning-based methodology to dynamically optimize the cutting parameter for a band saw capture the main trend of the health index and robustness to noise.

Table 2. The results of all methods under the two metrics.

\begin{tabular}{|l|l|l|}
\hline Method & MAE & RMSE \\
\hline RBF SVR & 0.216 & 0.250 \\
\hline Random Forest & 0.253 & 0.297 \\
\hline Neural Network & 0.159 & 0.219 \\
\hline CNN & $\mathbf{0 . 0 6 7}$ & $\mathbf{0 . 0 8 4}$ \\
\hline
\end{tabular}

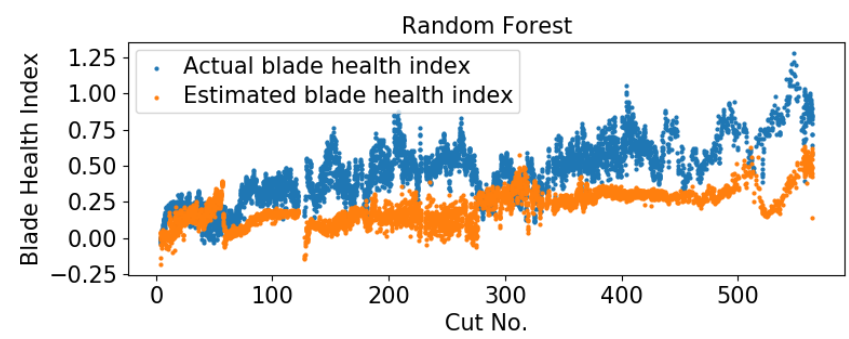

(b)

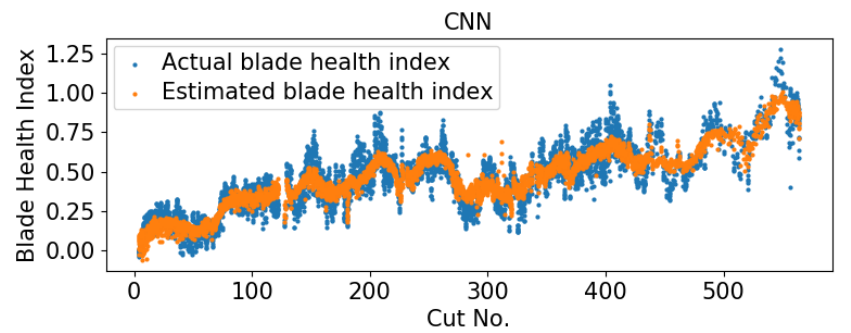

(d) machine. The significant blade degradation mitigation result demonstrates the effectiveness of the proposed methodology. The comparison of results show that the CNN performed better in mapping the complex relatiohship compared to traditional machine learning models. The future research work in this area will include setting up an experiment to measure the performance of the developed cutting parameter optimization method compared to an experienced machine operator. 


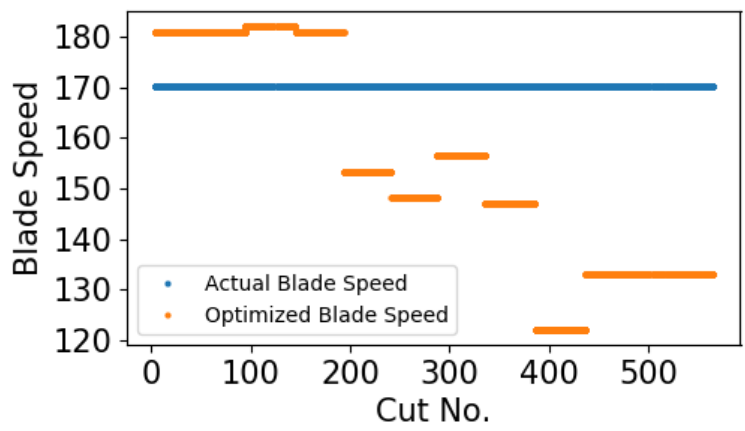

(a)

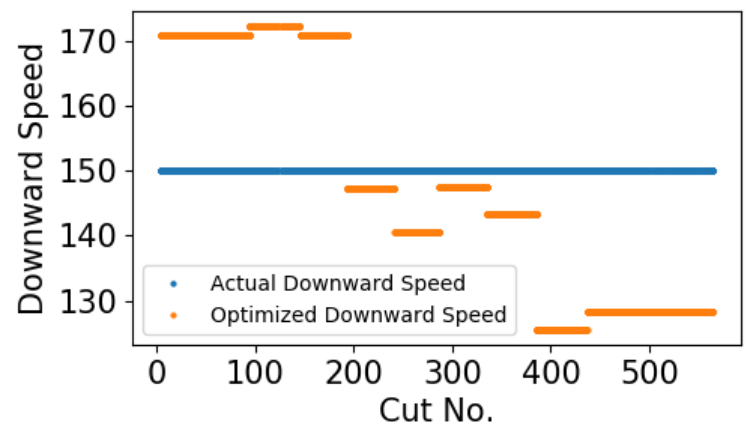

(b)

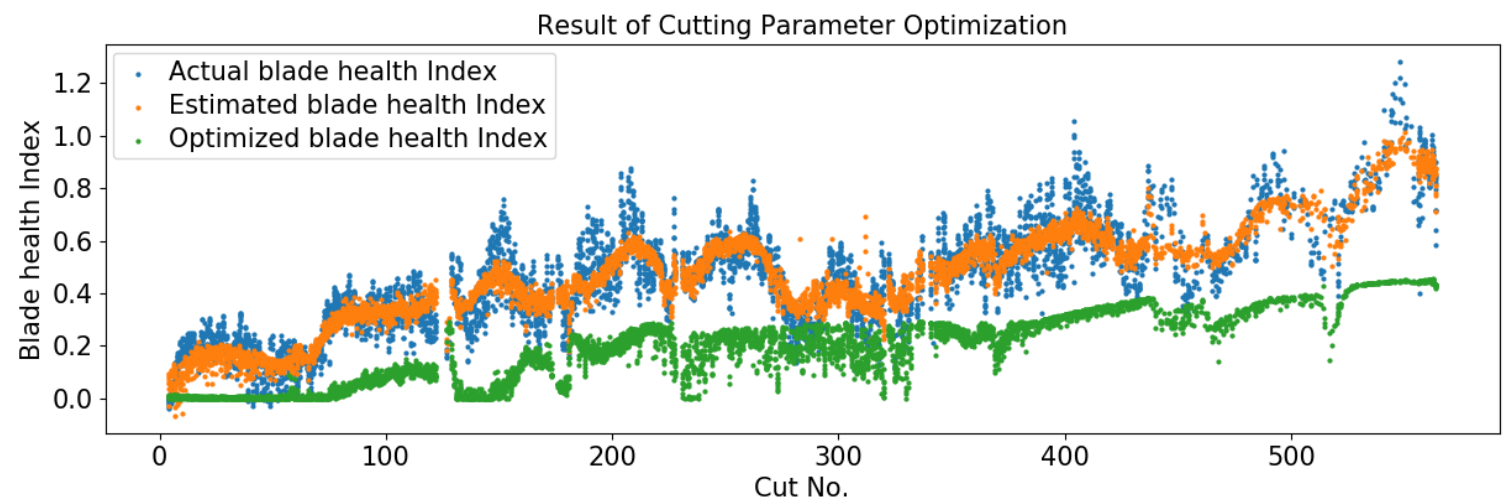

(c)

Figure 6. Results of cutting paramter optimization. (a) blade speed optimization, (b) downward speed optimization, (c) health index optimization.

\section{REFERENCE}

Asiltürk, I., \& Ünüvar, A. (2009). Intelligent adaptive control and monitoring of band sawing using a neural-fuzzy system. Journal of Materials Processing Technology, 209(5), 2302-2313. https://doi.org/10.1016/j.jmatprotec.2008.05.031

Bhattacharya, A., Das, S., Majumder, P., \& Batish, A. (2009). Estimating the effect of cutting parameters on surface finish and power consumption during high speed machining of AISI 1045 steel using Taguchi design and ANOVA. Production Engineering, 3(1), 31-40. https://doi.org/10.1007/s11740-008-0132-2

Camposeco-Negrete, C. (2015). Optimization of cutting parameters using Response Surface Method for minimizing energy consumption and maximizing cutting quality in turning of AISI 6061 T6 aluminum. Journal of Cleaner Production, 91, 109-117. https://doi.org/10.1016/j.jclepro.2014.12.017

Jia, X., Di, Y., Feng, J., Yang, Q., Dai, H., \& Lee, J. (2018). Adaptive virtual metrology for semiconductor chemical mechanical planarization process using GMDH-type polynomial neural networks. Journal of Process Control, 62 , https://doi.org/10.1016/j.jprocont.2017.12.004

Jia, X., Huang, B., Feng, J., Cai, H., \& Lee, J. (2018). A Review of PHM Data Competitions from 2008 to 2017 Review of PHM Data Competitions from 2008 to 2017 : Methodologies and Analytics. (September), 0-10.

Jia, X., Jin, C., Buzza, M., Di, Y., Siegel, D., \& Lee, J. (2018). A deviation based assessment methodology for multiple machine health patterns classification and fault detection. Mechanical Systems and Signal Processing, 99, 244-261. https://doi.org/10.1016/j.ymssp.2017.06.015

Jia, X., Zhao, M., Di, Y., Li, P., \& Lee, J. (2018). Sparse filtering with the generalized $1 \mathrm{p} / 1 \mathrm{q}$ norm and its applications to the condition monitoring of rotating machinery. Mechanical Systems and Signal Processing, 102 , $198-213$ https://doi.org/10.1016/j.ymssp.2017.09.018

Jing, L., Zhao, M., Li, P., \& Xu, X. (2017). A convolutional neural network based feature learning and fault diagnosis method for the condition monitoring of gearbox. Measurement: Journal of the International Measurement Confederation, 111, 1-10. https://doi.org/10.1016/j.measurement.2017.07.017 
Li, J., Mei, X., Prokhorov, D., \& Tao, D. (2017). Deep Neural Network for Structural Prediction and Lane Detection in Traffic Scene. IEEE Transactions on Neural Networks and Learning Systems, 28(3), 690-703. https://doi.org/10.1109/TNNLS.2016.2522428

Li, P., Jia, X., Feng, J., Davari, H., Qiao, G., Hwang, Y., \& Lee, J. (2018). Prognosability study of ball screw degradation using systematic methodology. Mechanical Systems and Signal Processing, 109, 45-57. https://doi.org/10.1016/j.ymssp.2018.02.046

Li, P., Jia, X., Sumiya, M., Kamaji, Y., Ishiguro, M., Pahren, L., \& Lee, J. (2019). A Novel Method for Deposit Accumulation Assessment in Dry Etching Chamber. IEEE Transactions on Semiconductor Manufacturing, PP(c), 1-1. https://doi.org/10.1109/tsm.2019.2904889

Lughofer, E., \& Pratama, M. (2018). Online Active Learning in Data Stream Regression Using Uncertainty Sampling Based on Evolving Generalized Fuzzy Models. IEEE Transactions on Fuzzy Systems, 26(1), 292-309. https://doi.org/10.1109/TFUZZ.2017.2654504

Mewada, B., \& Raval, H. (2016). Optimization of cutting rate and taper in bandsawing process using TAGUCHI and Desirability Approach. VIII(2).

Qiao, G., Liu, G., Ma, S., Wang, Y., Li, P., \& Lim, T. C. (2019). Thermal characteristics analysis and experimental study of the planetary roller screw mechanism. Applied Thermal Engineering, 149(December 2018), 1345-1358. https://doi.org/10.1016/j.applthermaleng.2018.12.137

Saglam, H. (2011). Tool wear monitoring in bandsawing using neural networks and Taguchi's design of experiments. International Journal of Advanced Manufacturing Technology, 55(9-12), 969-982. https://doi.org/10.1007/s00170-010-3133-1

Sangwan, K. S., Saxena, S., \& Kant, G. (2015). Optimization of machining parameters to minimize surface roughness using integrated ANN-GA approach. Procedia CIRP, 29 , 305-310. https://doi.org/10.1016/j.procir.2015.02.002

Sarikaya, M., \& Güllü, A. (2015). Multi-response optimization of minimum quantity lubrication parameters using Taguchi-based grey relational analysis in turning of difficult-to-cut alloy Haynes 25. Journal of Cleaner Production, 91, 347-357. https://doi.org/10.1016/j.jclepro.2014.12.020

Yang, S. (2016). An adaptive prognostic methodology and system framework for engineering systems under dynamic working regimes. University of Cincinnati. 